\title{
Off the Air
}

\section{Edmun B. Richmond}

This month, I want to discuss practical antennas which can be erected for shortwave listening. These antennas are inexpensive to build and easy to install. They can be used at a home site or at a school location. All of the antennas which are described are wire antennas, nothing fancy, but very effective.

There are two basic kinds of antennas, those for general coverage of the shortwave spectrum, and those which cover a particular bandwidth. The old axiom of making the antenna as long as possible and getting it up as high as possible certainly hold true for the general coverage antenna. The optimum length for this antenna is 740 feet. The specialized frequency antennas will have a length according to the desired frequency. In general, the higher off the ground, the better the reception.

To make these antennas, you will need the following material, which can be purchased at any radio supply house or electrical supply company. The wire for the antenna should be number 14 or number 12 solid copper. This can be purchased as either bare or with an insulated jacket. The only advantage of the insulation is that it protects the antenna during ice storms. With ice freezing over the entire antenna system, you will not be able to hear much, since the ice shorts out the antenna so that not much signal reaches the receiver. You will need three egg insulators, and a length of feedline which connects the antenna to the receiver.

Now, let's look at the configuration of the antenna. Wire antennas can be fed either with the feedline in the center of the antenna, or at the end of the antenna. But before deciding what type of antenna/feedline you are going to use, you have to determine what number of antenna terminals are on the back of your receiver. Your receiver will have either one of two terminals to be connected to the antenna. If you have only one terminal, you can use the same number 14 or 12 copper wire stock that you used as the antenna. If your receiver has two terminals, then you may use any correct length of $300 \mathrm{ohm}$ tv twinlead.

Here is an example of a center-fed general coverage antenna:

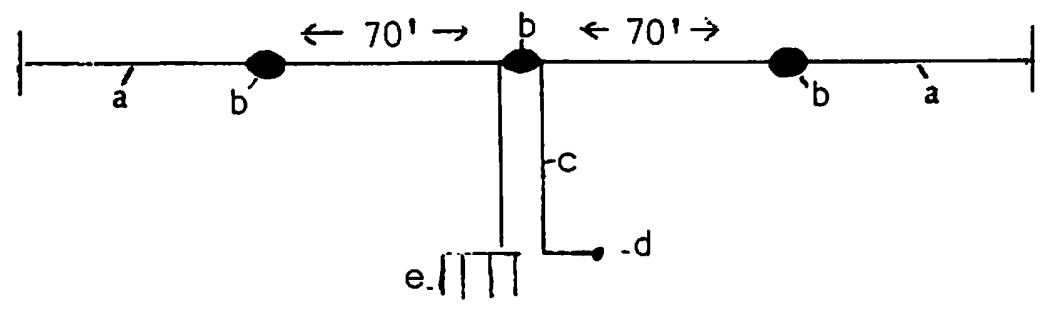
a. clothesline
b. insulator
c. $300 \mathrm{OHM}$ twinlead
d. Receiver
e. ground 
The tools needed to construct the antenna are a knife, wire cutters, pliers, solder, and a soldering gun. Here's step-by-step instructions on how to make this antenna:

1- Cut two equal lengths of wire, each $70^{\prime} 6^{\prime \prime}$ (the extra length of six inches is to wrap the egg insulator around the wire)

2- With your knife, scrap the lacquer insulation off the bare wire (or the plastic jacket) to a length of 4 inches. Do this to the four ends of the lengths of wire you cut.

3- Take one of the lengths of wire. Insert one end of the scraped wire through the egg insulator, wind it around the groove, and then back around the length of wire. Tighten with pliers, then solder the connection. Do the same with the second insulator on the opposite end of that length of wire. Take the other length of wire and install the third insulator on one end as before.

4- Now take the other end of the wire (the one without an insulator), and pass it through one of the insulators of the wire which has the two insulators. Solder as before.

5- Take your feedline, cut away about two inches of insulation, and wrap one side of the feed just to the left of the center insulation. Take the other side and wrap it to the right of the center insulator. Solder both connections.

That completes your antenna. Now you have to hang it. Find two trees, poles, or buildings which are far enough apart for you to hang the antenna between them. Use a length of plastic clothes line to fasten the antenna to the tree. Place one end of the clothes line through the other hole of the egg insulator and wrap it in the other set of grooves in the insulator. Make a knot and tie. Then run the length of the clothesline to the tree. Mount your antenna as high as possible by tying each length of clothesline to whatever is available. Now bring the feedline into your room where the radio is located. Cut away about an inch of the insulating material, and wrap one bare wire to each terminal on the receiver.

If your receiver has only one terminal for an antenna, you will make your version of this antenna the same length, but without the center insulator. With this antenna, you can tie your feedline to any point along the length of the antenna wire, which is convenient. You're in business for some fine reception. 
To finish up our amateur radio call sign allocations, here are those for German-speaking, Portuguese-speaking, and Russian-speaking countries.

German-Speaking

Austria- OE

Germany, East- Y2

Germany, West- DA-DL

Switzerland- HB

\section{Russian-Speaking}

A complicated system depending on the region and constituent Soviet Republic; whether a private station or club station. All Russian prefixes begin with the letter $U$, plus another letter and number.

Next time, l'll give you antenna dimensions for specific bandwidths, as well as some other configurations for general coverage antennas. We'll also talk about antenna directivity. Until then, good listening.

\section{Portuguese-Speaking}

Brazil- PP,PT,PY

Portugal- CT

\section{Mis \\ The Modern Language Journal}

.. . a quarterly journal devoted primarily to methods, pedagogical research, and lopics of professional interest to all language teachers.

\section{EDITOR: David P. Benseler \\ 314 Cunz Hall \\ The Ohio State University \\ Columbus, Ohio 43210}

Subscription Rates:

Institutions: S20/year

Individuals: \$12/year

Foreign postage: \$\$/year.

including Canada and Mexico
Send subecriptions to:

Journal Division

University of Wisconsin Prest

114 North Murray Street

Madison, Wisconsin 53715 U.S.A. 